\title{
EDUCACIÓN LÚDICA: \\ UNA OPCIÓN DENTRO DE LA EDUCACIÓN AMBIENTAL EN SALUD. SEGUIMIENTO DE UNA EXPERIENCIA RURAL COLOMBIANA SOBRE LAS GEOHELMINTIASIS
}

\author{
Nohora Aydée Ramírez Sánchez, Antropóloga, Ph.D. ${ }^{1}$, María Del Pilar Díaz Murillo, Antropóloga. M.Sc. ${ }^{* *}$, \\ Patricia Reyes Harker, MD. M.Sc. ${ }^{3}$ Y Orlando Cueca GonzÁlez, diseÑador INDUSTRIal ${ }^{4}$ \\ ${ }^{1}$ Doctorada en Educación con énfasis en Mediaciones Pedagógicas, Profesora, Facultad de Ciencias Económicas y Sociales, \\ Programa de Trabajo Social, Universidad De La Salle, Grupo interinstitucional de Antropología Médica Facultad de Medicina, \\ Universidad Nacional de Colombia; ${ }^{2}$ M.Sc. Salud Pública, Profesora Asociada, Departamento de Salud Pública, \\ Grupo interinstitucional de Antropología Médica Facultad de Medicina, Universidad Nacional de Colombia, Bogotá, Colombia. \\ ${ }^{3}$ M.Sc. Medicina Tropical, Profesora Asociada, Departamento de Salud Pública, Facultad de Medicina, Universidad Nacional de Colombia, Bogotá. \\ ${ }^{4}$ Diseñador Industrial, Universidad Nacional de Colombia, Bogotá.
}

\begin{abstract}
Resumen
Las geohelmintiasis son un problema de Salud Pública en los países en desarrollo, especialmente en escolares y preescolares, su control se basa en la integración de varias estrategias entre las cuales es esencial la educación en salud, que por sí sola ha demostrado ser altamente costo-efectiva. En este artículo se publican los resultados correspondientes a un estudio cualitativo aplicado, etnográfico, con enfoque crítico y participación comunitaria, cuyo objetivo fue desarrollar y hacer un seguimiento a una experiencia de educación lúdica, ambiental, dirigida a la prevención y el control de las geohelmintiasis en "La Virgen" (Cundinamarca, Colombia), basado en tres elementos: número de participantes en las actividades lúdicas, aceptación de las mismas y dificultades en su aplicación. Se efectuó un muestreo intencional a través del cual se seleccionaron maestros y estudiantes entre los ocho a quince años de cuatro instituciones educativas. Las técnicas utilizadas fueron entrevistas, observación participante, diarios de campo e instrumentos que permitieran el seguimiento de cada actividad. La validez de la metodología estuvo dada por la triangulación de datos. Las actividades lúdicas desarrolladas contaron con aceptación por parte de escolares, maestros y padres de familia. Hubo una fácil comprensión de los objetivos de los juegos y demás actividades lúdicas. Se presentó una participación considerable en el proceso por parte de maestros, alumnos, padres de familia y líderes comunitarios. Los primeros (especialmente las ludotecarias) se mostraron animados a colaborar en las actividades lúdicas y a emplearlas a futuro en sus clases; además, solicitaron otras capacitaciones relacionadas con salud. A partir de la interacción de la gente con el equipo de la Universidad Nacional surgieron nuevos materiales y actividades educativas lúdicas en salud (carteleras, canciones y un festival de la salud). Esta experiencia educativa ambiental lúdica permitió corroborar que la educación involucra un proceso profundo de enseñanza aprendizaje donde se construyen nuevos saberes; no se debe reducir a un nivel informativo que se refiere a una transmisión de datos emisor-receptor con carácter jerárquico. La lúdica optimiza el aprendizaje, promueve la participación y la creatividad. Además, permite identificar fácilmente problemas ambientales sentidos por la gente y aspectos relativos a la prevención que deben trabajarse tanto a nivel de saneamiento básico (por ejemplo, ausencia de alcantarillado) como de educación en salud, estimulando a la población a mejorar su calidad de vida.
\end{abstract}

Palabras clave: educación ambiental, educación lúdica, participación comunitaria, helmintiasis, escolares

Recibido: Marzo 3 de $2011 \quad$ Aceptado: Mayo 25 de 2011

* Dirección de correspondencia: María del Pilar Díaz, mddiazm@unal.edu.co y mpdiazm@gmail.com. Dirección postal: Carrera 30 No. 45-03, Bogotá, Colombia Departamento de Salud Pública, Facultad de Medicina, Oficina 143, Universidad Nacional de Colombia. 


\title{
Play-based education: an option in ENVIRONMENTAL EdUCATion IN HEALth. MONITORING OF RURAL COLOMBIAN EXPERIENCE ON GEOHELMINTHIASIS
}

\begin{abstract}
Soil-transmitted helminths are a public health problem in developing countries, especially in school and preschool children. Their control is based on the integration of various strategies such as health education, which alone has proven to be highly cost-effective. This paper describes a qualitative applied study with an ethnographic design, a critical approach, and community participation. The study objective was to develop and track a play-based and environmental learning experience, aimed to prevent and control soil-transmitted helminths in "La Virgen" (Departamento de Cundinamarca, Colombia). The track was based on three elements: number of participants in recreational activities, acceptance of these activities, and difficulties in their implementation. Purposive sampling was performed through which teachers, and students aged 8 to 15 , were selected mainly from 4 educational institutions. The techniques used were interviews, participant observation, field notes, and other instruments that allow the monitoring of each activity. The validity of the methodology was described by the triangulation of data. Recreational activities were accepted by school teachers and parents. The objectives of the games and other fun activities were easily understood by the children and other participants. Teachers, students, parents and community leaders also became involved in the process. The teachers, especially the toy librarians, were encouraged to attend recreational activities and promote their future employment in their classes. These instructors also requested additional health training workshops from the researchers. Effective collaboration between the participants from La Virgen and the researchers from la Universidad Nacional produced new materials and recreational activities in health education (such as billboards, songs, and a health festival). This educational experience corroborates the findings of previous studies, which indicate that simply presenting information does not necessarily ensure that it will be internalized by the subjects. Play-based education optimizes learning, while encouraging participation and creativity. In addition, it allows for the easy identification of environmental problems felt by the population, and the development of preventative activities related to both basic sanitation (e.g. lack of sewerage) and health education. These aspects of the project have the ability to improve participants' quality of life.
\end{abstract}

Key words: environmental education, play-based education, community participation, helminthiasis, school children

\section{EDUCAÇÃo LÚDICA: UMA OPÇÃO EM EDUCAÇÃO EM SAÚDE AMBIENTAL. MONITORAMENTO DE EXPERIÊNCIA COLOMBIANA RURAL EM GEOHELMINTOSES}

\section{Resumo}

As geohelmintoses são um problema de saúde pública nos países em desenvolvimento, especialmente nos escolares e pré-escolares, seu controle é baseado na integração de várias estratégias, entre os quais é essencial a educação para a saúde, que por si só tem provado ser altamente custo-eficaz. Este artigo publicou resultados de um estudo qualitativo etnográfico aplicada, com abordagem crítica e participação da comunidade cujo objetivo era desenvolver e acompanhar uma experiência de aprendizagem lúdica, ambiental, visando a prevenção e controle das helmintoses transmitidas pelo solo em "La Virgen" (Cundinamarca, Colômbia), com base em três elementos: número de participantes em atividades de recreação, aceitação das mesmas e as dificuldades de implementação. Foi realizada uma amostragem intencional através do qual professores e alunos de 8 a 15 anos foram selecionados em quatro escolas. As técnicas utilizadas foram entrevistas, observação participante, diários de campo e ferramentas que permitem a monitorização de cada atividade. A validade da metodologia foi dada pela triangulação de dados. As atividades lúdicas contaram com aceitação dos estudantes, professores e pais. Houve uma compreensão fácil dos objectivos de jogos e outras actividades recreativas. Houve uma participação significativa no processo de professores, alunos, pais e líderes comunitários. Os primeiros (especialmente "ludotecarias") foram encorajados a colaborar em actividades recreativas para seu emprego futuro em suas classes; além disso, solicitaram outros treinamentos relacionados à saúde. A partir da interação das pessoas com a equipe da Universidade Nacional vieram novos materiais e atividades recreativas de educação em saúde (cartazes, 
músicas, e um festival de saúde). Esta experiência educativo ambiental lúdica corroborou que a educação envolve um profundo processo de ensino e de aprendizagem onde o conhecimento novo é construído; não pode ser reduzida a um nível de informação que se refere a um transmissor-receptor de dados com carater hierárquico. A lúdica otimiza o aprendizado, promove a participação ea criatividade. Além permite a fácil identificação de problemas ambientais sentida pelo povo e os aspectos de prevenção para ser trabalhado tanto no saneamento básico (por exemplo, falta de esgoto) e educação em saúde, incentivando as pessoas a melhorar a sua qualidade de vida .

Palavras-chave: educação ambiental, educação lúdica, participação comunitária, helmintíase, escolares

\section{Introducción}

En los países en vía de desarrollo las geohelmintiasis son un problema de salud pública que fundamentalmente afecta a los escolares y preescolares. Aproximadamente en el mundo hay 2000 millones de personas infectadas con helmintos, de éstas por lo menos 300 millones sufren morbilidad severa asociada como anemia, desnutrición crónica y mal desempeño escolar (1-3).

Dentro de las estrategias recomendadas para la prevención y el control de las geohelmintiasis, además de la desparasitación periódica con antihelmínticos, son indispensables la educación en salud y la ambiental con participación comunitaria para motivar comportamientos saludables, que reduzcan las tasas de reinfección por geohelmintos en los individuos de las comunidades afectadas por estos parásitos intestinales. Una estrategia recomendable para la educación en salud es articular el conocimiento de los diferentes tópicos sobre los parásitos intestinales al plan de estudios escolar mediante actividades lúdicas, que conduzcan a los estudiantes a adquirir dicho conocimiento a la vez se constituyen en agentes multiplicadores de éste y de la prevención de tales infecciones.

En cuanto a la prevalencia de los geohelmintos en los escolares de la Inspección de La Virgen, área de este estudio, en el año 2005 era de $27,7 \%$ para Ascaris lumbricoides, $21,2 \%$ para Trichuris trichiura y de $7,1 \%$ para Uncinaria $s p(4)$.

Por otra parte, en un estudio sobre conocimientos, actitudes y prácticas ( $\left.\mathrm{CAP}^{\prime} \mathrm{s}\right)$ relativas a las geohelmintiasis en esa misma población, se evidenció la importancia de realizar actividades de educación ambiental. Por ejemplo, se estableció que un $60.8 \%$ de los cuidadores de niños, en una muestra de 130 personas, señaló que los niños, cuando están en casa, usualmente van al campo a hacer sus necesidades. Pero cuando están en la escuela, un $81,5 \%$ de los cuidadores de los niños indica que utilizan las instalaciones sanitarias. Sin embargo, mediante observación se advirtió que los baños tanto de las escuelas como de las casas eran inadecuados y que la mitad de las viviendas contaban con tazas sanitarias o similares, pero no en las mejores condiciones. Ante esas circunstancias es frecuente que la gente haga sus deposiciones a campo abierto (5).

Igualmente, en ese mismo estudio, se estableció que los padres de los niños creen que el Ascaris lumbricoides al salir del cuerpo muere y no constituye un riesgo para salud, e incluso, ellos y sus hijos, "...sostienen que las lombrices sirven de abono para las plantas o de alimento para las gallinas. Estas creencias sumadas a la falta de instalaciones sanitarias adecuadas, explicarían por qué en esta región aún existen altas prevalencias de parásitos intestinales incluidos los geohelmintos..." (5).

Los resultados del estudio CAP's en La Virgen sustentaron la necesidad de un acompañamiento educativo para su población, no sólo dirigido al cambio de hábitos, como control de factores de riesgo, sino a generar conciencia de su realidad y de cómo lo que sucede en su ambiente socio-natural tiene que ver con su salud; en este caso con la presencia de las geohelmintiasis. Hay que tener presente que el fundamento de la educación está relacionado con el acontecer del acto en la vida cotidiana, la cual a su vez da sentido a la existencia, no así "los conocimientos, la información, ni las verdades transmitidas a través de discursos y consignas" (6).

Para adentrarse en esa cotidianeidad es necesario realizar nuevas acciones educativas que desarrolladas mediante la lúdica, como las del presente trabajo, ayudan a mejorar la calidad de vida de la gente. Ello implica construir un mundo más sano, en una cotidianidad, que relaciona lo personal y lo grupal, ambos orientados a la toma de conciencia ambiental y ciudadana, la cual se logra sólo si se encuentra apoyada por una población organizada y consciente de sus derechos, obligaciones y de su interacción con el ambiente al cual pertenecen. 
Dada la importancia de la educación lúdica en salud como una opción en la formación ambiental en salud, el presente artículo describe el seguimiento hecho a una experiencia de educación lúdica, ambiental, dirigida al control de las geohelmintiasis en la Inspección de La Virgen, población rural colombiana, con base en tres parámetros de valoración: número de participantes en las actividades lúdicas educativas, aceptación de las mismas y dificultades en su aplicación.

\section{La educación lúdica y la educación en salud}

Tanto la educación en salud como el saneamiento ambiental son esenciales en la prevención y el control de las geohelmintiasis. Asaolu y Ofoezie concluyeron que el saneamiento básico y la educación en salud reducen considerablemente las prevalencias y la intensidad de las helmintiasis (7).

Es de resaltar que el concepto de promoción en salud sobrepasa al de educación en salud, por ser un proceso más amplio e integral, que incluye la participación de toda la población en el contexto de la vida cotidiana, el cual trasciende el estimar el riesgo de enfermar. Dicha noción se basa en un concepto ampliado de salud, que considerado como un estado positivo y dinámico de búsqueda de bienestar comprende, en interacción, las dimensiones física, personal/emocional (autorrealización personal y afectiva), ambiental (adaptación al ambiente) y social (equidad y vulnerabilidad de diversa índole).

Aunque hay varios abordajes de la educación en salud, aquí sólo se mencionan los que se consideran más relevantes, incluyendo "la educación lúdica", la cual se seleccionó porque conlleva a la participación, el disfrute y la creatividad a partir de la vida cotidiana. La educación lúdica de acuerdo a Nunes de Almeida tiene como objetivos "... la estimulación de las relaciones cognoscitivas, afectivas, verbales, psicomotoras, sociales, la mediación socializadora del conocimiento y la provocación de una reacción activa, crítica [y] creativa..." de las personas en pro de su bienestar (8). En ese sentido, como señaló Froebel en el siglo XIX, citado por Nunes de Almeida, la pedagogía debe considerar al niño como sujeto creador y éste, debe despertar por medio de estímulos, sus facultades propias para la creación productiva. Además, Froebel consideró que los juegos eran decisivos en la socialización de los niños (8).

Asimismo, la lúdica se constituye en el elemento catalizador de la participación, ya que "los conocimientos, informaciones y actitudes en salud dependen mucho menos de la sofisticación de los métodos de comunicación o de la complejidad de las técnicas pedagógicas y mucho más de la participación directa de la población en la definición de sus necesidades y en la selección de los caminos más adecuados a su proyecto de vida" (9).

Además, la educación en la escuela mediante actividades lúdicas "... es más bien una actitud, una predisposición del ser frente a la vida, frente a la cotidianidad. Es una forma de estar en la vida, y de relacionarse con ella en esos espacios cotidianos en que se produce disfrute, goce, acompañado de la distensión que producen actividades simbólicas e imaginarias como el juego, la chanza, el sentido del humor, el arte y otra serie de actividades (...) que se producen cuando interactuamos sin más recompensa que la gratitud que producen dichos eventos" (10).

El juego, el disfrute, como parte de la lúdica constituyen una forma agradable de obtener una mayor participación de la gente porque "jugando los niños [y adultos] toman conciencia de lo real, se implican en la acción, elaboran razonamientos y juicios. (...). Marginar el juego de la educación equivaldría a privarla de uno de sus instrumentos más eficaces, por ello el educador debe asegurar que la actividad del niño o la niña sea una de las fuentes principales de su aprendizaje y desarrollo, pues a través de la acción y la experimentación, ellos expresan sus intereses, sus motivaciones y descubren las propiedades de los objetos, relaciones, etc." (11).

El juego desarrolla diferentes habilidades, contribuye a conformar redes de cooperación y ayuda, a afrontar situaciones que se presentan en la vida real, “...descubre el valor del "otro" por oposición a sí mismo, e interioriza actitudes, valores y normas que contribuyen a su desarrollo afectivosocial y a la consecución del proceso socializador (...)" (11). Estas características del juego y de lo lúdico permiten en la educación en salud trascender de la mera enfermedad a las condiciones de vida, que es uno de los propósitos a mediano y corto plazo de este trabajo. Valga resaltar que los juegos como parte de lo lúdico son importantes porque no sólo dan placer a los participantes sino que rescatan la condición de ser niño, facilitan la interacción social y constituyen un espacio democrático para la libre expresión (12). Es importante que los maestros integren los juegos y otros materiales lúdicos como mediaciones pedagógicas en su actividad académica, entendidas, en términos de Gutiérrez y Prieto, "...como el tratamiento de los contenidos y de las formas de expresión de los diferentes temas a fin de hacer posible el acto educativo, dentro del horizonte de una educación concebida como participación, creatividad, 
expresividad y relacionalidad (...) en su actividad académica" (13).

Continuando con los enfoques, uno de los más utilizados en la educación en salud se basa en el cambio de comportamientos, con el cual se espera que "los conocimientos", conduzcan a la adopción de estilos de vida saludables. Sin embargo, no tiene en cuenta los significados que esos "malos hábitos" podrían tener para las personas; se adopta una actitud controladora cuyo objetivo es trazado desde fuera de la comunidad, sin pensar en los proyectos de vida de la gente (9). Por tanto, este es un enfoque de carácter unidireccional y vertical, que da prelación a las prioridades establecidas por los servicios o los profesionales de salud sin tener en cuenta las características propias de las comunidades con las que se actúa.

La visión de cambio comportamental ha sido identificada dentro de la perspectiva conductista. Fundamentalmente, ésta es concebida en el saber y la jerarquía que muestra el maestro en el aula de clase, él se atribuye la dirección del desarrollo de la actividad; en tanto el estudiante puede ser visto como un ser que absorbe, retiene y reproduce lo dado, se amolda a un sistema impositivo y avasallador. Corresponde a lo que Paulo Freire llamó la "educación bancaria" porque "la educación" equivale a un depósito de información en los alumnos (12). No se construye con ellos; no se orienta a una toma de conciencia sobre lo que implica un ambiente saludable.

También han surgido otras posiciones, identificadas como horizontales y dialógicas, que buscan algún nivel de reciprocidad de la gente. Ellas son conocidas como ejemplos de comunicación en grupos y participativa (14).

Mientras el primer modelo implica una consideración sobre el mensaje, en el segundo "cada integrante del grupo se constituye a la vez en emisor y receptor para convertirse en coautor de los mensajes, el sujeto entonces es un reproductor del mensaje central que es reelaborado conjuntamente" (14). No obstante, esos modelos (la educación bancaria y el dialógico) se identifican porque desarrollan un mayor énfasis en los conceptos, las necesidades, contenidos y métodos que en los medios utilizados. Las personas favorecidas establecen los objetivos de acuerdo con sus necesidades, pero dependen de la guía del facilitador (14). Se nota pues que, la orientación de tales modelos brinda algún nivel de autonomía a los sujetos y a las comunidades porque permite a las poblaciones fijar sus prioridades.
Frente a una educación que condiciona se encuentra otra problematizadora, cuyo origen se remonta a lo movimientos de educación popular de la década del 50 del siglo pasado. Este enfoque reconoce que educadores y alumnos son afectados por la realidad que aprenden; tal aprendizaje permite tomar conciencia sobre aquélla y transformarla (12).

La educación problematizadora de Freire, de carácter horizontal, se consideró pertinente para el presente trabajo porque, como afirman Lara y Torres, emplea los asuntos de salud para motivar y concientizar a la población general y a educadores y educandos sobre la relevancia de un trabajo que incorpore salud, ambiente y ciudadanía (15). Este enfoque, utilizado en un trabajo para el control de la esquistosomiasis, consiguió que maestros y alumnos perfeccionasen sus conocimientos sobre la patología, sus habilidades metodológicas y su confianza para mejorar su calidad de salud. (15)

Por otra parte, la perspectiva crítica propia de este enfoque tiene la ventaja de estimular la participación comunitaria y a través de ella permitir que las personas se den cuenta que no son las únicas afectadas por un determinado problema y que comparten muchas cosas en su diario vivir. Además, tienen la oportunidad de mirarse a sí mismas y a su cotidianidad de manera crítica, a partir de lo cual construyen un conocimiento propio, revisan su historia y la relacionan con el medio ambiente y la salud. En ese sentido identifican los principales problemas vinculados con estos aspectos, a la vez que construyen nuevas relaciones desde una perspectiva sostenible y sustentable.

Igualmente, el enfoque de la educación problematizadora permite que en el proceso socializador maestro y alumno aprendan mediante el diálogo reflexivo que establecen acerca de un tema específico. "La construcción del conocimiento se dará en función de la reflexión que no deberá ser una mera abstracción. El Hombre, siempre deberá ser comprendido en relación a su vínculo con el mundo" (16) porque, además de vivir en él, adquiere conocimiento sobre éste, a la par que lo convierte en un conocimiento práctico.

Este tipo de educación problematizadora es deseable en poblaciones con una propensión a la pasividad, como la de La Virgen (actitud identificada en el estudio previo sobre CAP's) (5), porque se centra en que la misma población desarrolle acciones a partir de su conocimiento reflexivo, con el fin de transformar sus condiciones de vida y, por ende, su futuro. Por lo demás, "la tarea de la 
educación no es decirles [a otros] lo que es importante" (9), tampoco se limita a transmitir conocimiento, como antes se mencionó, sino a facilitar las circunstancias para que la misma comunidad exprese lo que es relevante para ella y sean apoyados en sus actividades de cambio.

Giroux, en 1988, señaló respecto a los docentes que emplean la educación problematizadora que aunque a menudo critican las formas dominantes de conocimiento eso no significa que tengan que quedarse en la crítica sino que "deben centrarse en la generación de conocimientos que ofrezcan posibilidades concretas de empoderar a las personas. Para decirlo más específicamente, una pedagogía crítica [o problematizadora] necesita el lenguaje de la posibilidad" (17) y preparación para afrontar la incertidumbre. Ante todo, el pensamiento crítico es reflexivo y evaluativo; debe guiar a un juicio razonado.

En ese orden de ideas es importante ratificar la relevancia de una "educación en salud" más allá de la mera "instrucción" o de suministrar información. Para ello es necesario reconocer y como tal entender a la gente para trabajar con ella mediante un proceso auto reflexivo y crítico que modifique su realidad. De ahí la relevancia de la educación problematizadora.

Por otra parte, la educación en salud y la ambiental están íntimamente relacionadas pues comparten varios objetivos y medios. Como argumentó Spork "ambas tratan la interrelación de cuestiones de justicia social y la sustentabilidad ecológica" (17). Además, como afirmó Mogensen, las dos comparten la meta de desarrollar habilidades de pensamiento crítico en los estudiantes y el compromiso de generar en ellos la responsabilidad y la motivación para involucrarse en la solución de los problemas a que se vean avocados (17).

Además, es importante que la educación, sin importar la especificidad que ella tenga, propenda por que los estudiantes forjen una curiosidad científica, que sean capaces de hacerse preguntas, reflexionar sobre las condiciones y estructuras sociales en que viven y buscar respuestas acordes a sus necesidades y las de su ambiente natural y social. En esto radica el pensamiento crítico.

Por otra parte, la estrategia de escuela saludable se articula tanto con la educación en salud como con la ambiental, a través de los tres ejes que presenta Ernesto Durán: el pedagógico, el ambiental y el de acciones en salud y nutrición (18) desde un enfoque lúdico que despierte curiosidad, que sea creativo, recreativo y constructivo. El primer eje se refiere al desarrollo de conocimientos, habilidades y des- trezas, que se articulan con el currículum, permiten una vida más saludable y parte de las necesidades sentidas de la gente y del reconocimiento del ambiente que les rodea. El segundo incluye los ambientes físicos que deben promover la salud y los ambientes sociales (esencialmente la escuela y la comunidad) que deberán impulsar el trabajo en paz y armonía, la libertad de expresión y de ser escuchado, sin discriminación alguna. El tercero comprende la prevención, las acciones dirigidas a mejorar la nutrición, la orientación a niños, niñas y sus familias sobre necesidades específicas, la información acerca de derechos y deberes en salud, la afiliación a la seguridad social y el uso adecuado y oportuno de los servicios de salud.

Por otra parte, como lo señala Velásquez, el ambiente lúdico de aprendizaje va más allá de la pregunta ¿con qué? y se ocupa por el ¿cómo?, cuya característica principal es la flexibilidad del pensamiento creativo (19), con el soporte de materiales sencillos de elaborar como los juegos didácticos y fáciles de conseguir, como colores, papel, tarjetas, pinturas etc. Actualmente las tecnologías de información y comunicación también ofrecen una amplia gama de posibilidades de aplicaciones lúdicas, ellas estimulan el placer por el juego educativo y creativo, además de promover la libertad al constituirse el juego en una actividad de carácter voluntaria.

Debido a la relevancia de la escuela saludable se considera que al retomar el ámbito de la escuela para la implementación de las actividades educativas lúdicas para la prevención de las geohelmintiasis y la promoción de la salud se la fortalece o impulsa como núcleo de transformación del conocimiento y de la calidad de vida de la población; pero además, se estimula el aprendizaje mediante el reconocimiento de otro y el trabajo en grupo.

\section{Materiales y métodos}

El trabajo se realizó en la Inspección de La Virgen, localizada a $4^{\circ} 45^{\prime}$ de latitud norte y $74^{\circ} 32^{\prime}$ de longitud oeste, aproximadamente a $100 \mathrm{~km}$. al suroccidente de Bogotá, con una altitud de 1443 m.s.n.m., temperatura promedio de $22^{\circ}$ C, precipitación anual promedio de $1696 \mathrm{~mm} 3$ y una superficie de $124 \mathrm{Km} 2$. Su población, incluida la del casco urbano y las veredas, asciende aproximadamente a 2000 habitantes (4). La gente se dedica al jornaleo (trabajo pagado por día de labor, denominado jornal) en cultivos de café, caña de azúcar y cítricos. La Inspección cuenta con una institución educativa departamental conformada por siete sedes (dos en la cabecera y cinco veredales) y una ludoteca comunitaria de la Caja de Compensación de 
Colsubsidio. Se entiende por este tipo de ludoteca aquella que se constituye en un espacio social donde la gente de determinada población, particularmente niños, se reúne para aprender mediante técnicas lúdicas sobre diversos aspectos que afectan su calidad de vida para asi contribuir con herramientas que ayuden a mejorar las condiciones problemáticas que los afectan.

\section{Población y muestra}

La población del estudio estuvo constituida por estudiantes y profesores de las diferentes sedes, más la ludoteca del casco urbano de la Inspección de La Virgen. En este se encuentran un Colegio y una escuela, el primero con 232 alumnos, 7 profesores, uno por cada área de conocimiento, y un coordinador académico. En la segunda había 80 alumnos matriculados al momento del estudio, distribuidos en cinco cursos, cada uno con un profesor coordinador del mismo. Las demás escuelas (sedes) corresponden a la vereda Galilea, con 14 escolares matriculados y un docente; la de la vereda San Nicolás con 13 estudiantes y un docente; la de la vereda El Líbano con 11 alumnos y un docente; la de la vereda El Retiro con 30 estudiantes y dos profesores; la de la vereda Santa Cruz con 49 escolares y dos docentes. (Datos obtenidos de los registros de matrículas del año 2009, proporcionados por la Secretaría del Colegio).
Al momento del estudio la ludoteca contaba con dos ludotecarias, quienes trabajan con toda la población escolar de la Inspección, cuyas actividades son programadas por turnos (en promedio cada escuela asiste un día por semana a la ludoteca).

Se utilizó un muestreo por conveniencia mediante el cual se seleccionaron como foco principal las escuelas de dos veredas (Galilea y San Nicolás), la del casco urbano y la ludoteca comunitaria ubicada también en la cabecera de la Inspección porque ofrecieron condiciones favorables para la implementación de ciertas actividades. Sin embargo, para algunas de ellas se adicionaron escuelas de otras veredas: El Retiro, Santa Cruz y El Líbano (Tabla 1). Las actividades lúdicas efectuadas incluyeron maestros y niños entre 8 a 15 años de estas instituciones y en ocasiones adultos de las veredas.

\section{Técnicas de recolección de datos}

Las técnicas empleadas fueron entrevistas, observación participante, diarios de campo e instrumentos que permitieran el seguimiento de cada actividad de acuerdo con el número de participantes, su aceptación y dificultades en su aplicación. La validez de la metodología estuvo dada por la triangulación de datos, es decir la utilización de "diferentes fuentes e instrumentos de recolección de datos" (20).

Tabla 1. Actividad de acuerdo a lugar de participación

\begin{tabular}{|c|c|}
\hline Actividad & Lugares que participaron \\
\hline Cartografía Social & Escuela casco urbano de La Virgen, Veredas Galilea y San Nicolás \\
\hline Juegos (Gusarin Gusarán y Aprender Pescando) & $\begin{array}{l}\text { Preescolar, escuela y ludoteca ubicadas en el casco urbano de La Virgen y } \\
\text { veredas Galilea, San Nicolás }\end{array}$ \\
\hline $\begin{array}{l}\text { Otras actividades lúdicas (Fabricación de títeres y ejecución de la obra el } \\
\text { Reino de mi Barriga, globoflexia, jornadas de dibujos y cuenteria) }\end{array}$ & Escuela casco urbano La Virgen, veredas Galilea y San Nicolás \\
\hline $\begin{array}{l}\text { Conformación del Grupo Jóvenes Estudiantes Ayudando a Recrear } \\
\text { (JEAR) }\end{array}$ & Estudiantes del Colegio del casco urbano de La Virgen \\
\hline Capacitaciones en actividades lúdicas & $\begin{array}{l}\text { Ludoteca casco urbano, profesores del casco urbano y veredas Galilea y } \\
\text { San Nicolás }\end{array}$ \\
\hline $\begin{array}{l}\text { Capacitación en las geohelmintiasis y entrega de artículos sobre } \\
\text { educación en salud, ambiental y lúdica. }\end{array}$ & Colegio y escuelas de La Virgen, el Retiro, el Líbano y Santa Cruz \\
\hline Talleres y capacitaciones varias a escolares & Ludoteca, escuela y colegio casco urbano y escuelas Galilea y San Nicolás \\
\hline Taller de microscopía. & Colegio y escuela de La Virgen, San Nicolás y Galilea. \\
\hline $\begin{array}{l}\text { Diseño, conceptualización y definición del dispositivo K-kal para } \\
\text { disposición de excretas }\end{array}$ & Población infantil y adulta de la vereda Galilea \\
\hline Frutivegecarnavalito/Factor $S$ & $\begin{array}{l}\text { Ludoteca, colegio, escuela y población general del casco urbano de La } \\
\text { Virgen y niños y adultos de las veredas Galilea, San Nicolás, Santa Cruz, } \\
\text { El Retiro y El Líbano }\end{array}$ \\
\hline
\end{tabular}


Los criterios de inclusión en el estudio fueron: 1) Ser niños y jóvenes (mujeres y hombres) entre 8 a 15 años de edad estudiantes de las escuelas seleccionadas y de la ludoteca y 2) Contar con el consentimiento informado de los padres o los representantes legales y del propio niño en el caso de los mayores de 9 años

El criterio de exclusión fue: 1) No tener el consentimiento informado de padres o cuidadores.

\section{Diseño del estudio}

Trabajo cualitativo aplicado, etnográfico, con enfoque crítico y participación comunitaria dirigido a desarrollar una educación lúdica, ambiental, enfocada al control de geohelmintiasis.

\section{Consideraciones éticas}

Para este trabajo se tuvieron en cuenta las normas para la investigación de la resolución No. 008430 de 1993 del Ministerio de Salud de Colombia (21), de la Convención Internacional de los Derechos del Niño de 1989 y del Código del menor de 1991 (22). El proyecto fue aprobado por el Comité de Ética de la Facultad de Medicina de la Universidad Nacional de Colombia, sede Bogotá y presentado a los líderes comunitarios, trabajadores de la salud, padres y madres de familia, ludotecarias, maestros y autoridades escolares y municipales quienes también lo aprobaron.

\section{Resultados}

Se realizaron actividades lúdicas de diversa índole, fundamentalmente con la población escolar. Aunque, en algunas participaron madres, padres y líderes comunitarios. Las acciones se dirigieron a motivar la curiosidad científica, a familiarizarse con los factores de riesgo relacionados con las geohelmintiasis, a identificar problemas ambientales en La Virgen de acuerdo con la perspectiva de sus pobladores y a estimular su participación en el proceso y en la continuidad del mismo así como en la búsqueda de soluciones a las dificultades identificadas.

\section{Cantidad de participantes por actividad y relevancia de la misma}

Las actividades desarrolladas fueron: capacitaciones lúdicas mediante talleres de microscopia (con maestros y alumnos) para reconocimiento de parásitos y de cartografía social (con escolares y sus madres) con el fin de identificar problemas ambientales sentidos por la gente en La Virgen; capacitaciones en el uso de los juegos "Gusarín, Gusarán" y "Aprender Pescando" y otras tareas de educación lúdica ambiental a escolares a través cuenteria, representaciones de animales con bombas (globoflexia) y talleres de reflexión. También se efectuó de manera lúdica y participativa el diseño del dispositivo K-kal para disposición de excretas y un Carnaval comunitario denominado "Frutivegecarnavalito/Factor S (Salud)", organizado entre el personal de la ludoteca de Colsubsidio y el equipo de trabajo del Departamento de Salud Pública (Facultad de Medicina) de la Universidad Nacional de Colombia. El número de participantes por actividad se reporta en la Tabla 2.

Dentro de las actividades efectuadas es importante destacar algunos casos que muestran la participación e interés de la gente. Así se tiene en primera instancia la

Tabla 2. Participantes por actividad

\begin{tabular}{|c|c|}
\hline Actividad & Número participantes \\
\hline Cartografía Social & $\begin{array}{l}\text { Seis madres y } 22 \text { escolares entre seis a doce años, de } 3^{0} \text { a } 5^{\underline{0}} \text { de primaria, tanto del casco } \\
\text { urbano como de las veredas San Nicolás y Galilea. }\end{array}$ \\
\hline Otras actividades lúdicas & 70 estudiantes de ambos géneros \\
\hline Conformación del Grupo JEAR & Seis jóvenes \\
\hline Capacitaciones en actividades lúdicas & Siete maestros y dos ludotecarias \\
\hline $\begin{array}{l}\text { Capacitación en las geohelmintiasis y entrega de } \\
\text { artículos sobre educación en salud, ambiental y lúdica }\end{array}$ & Seis maestros (cuatro mujeres y dos hombres) del casco urbano y las veredas participantes \\
\hline Talleres y capacitaciones varias a escolares & Entre 80 a 100 niños de las instituciones participantes \\
\hline Taller de microscopia & $\begin{array}{l}\text { Tres maestros (Casco urbano de La Virgen y veredas Galilea y Santa Cruz) y quince alumnos } \\
\text { (ocho niñas y siete niños, provenientes de veredas y el casco urbano) }\end{array}$ \\
\hline $\begin{array}{l}\text { Diseño, conceptualización y definición del dispositivo } \\
\text { K-kal para disposición de excretas }\end{array}$ & Doce adultos y doce niños(as) de la vereda Galilea \\
\hline
\end{tabular}


conformación y afianzamiento del Grupo Jóvenes Estudiantes Aprendiendo a Recrear (JEAR), integrado por muchachos (hombres y mujeres) líderes de la Inspección de La Virgen, quienes colaboraron en la organización del "Frutivegecarnavalito-Factor $\mathrm{S}$.

La capacitación a los maestros sobre parasitismo intestinal fue valiosa, pues todos consideraron factible replicarlas con sus alumnos mediante la utilización de las mediaciones pedagógicas aprendidas y transformadas con su participación. Cuatro indicaron formas lúdicas de hacerlas, otro tomaría la vía magistral y el último sólo mencionó que lo haría durante la clase de ciencias naturales. Afirmaron que las explicaciones habían sido claras, aunque dos educadores señalaron dificultades con los nombres científicos de los geohelmintos. Algunos docentes agregaron que les gustaría recibir capacitaciones acerca de otros temas también relacionados con salud.

Cuando se realizó el entrenamiento para el manejo de los juegos "Gusarín, Gusarán" y "Aprender Pescando", los maestros y ludotecarias comentaron que los juegos son buenos porque permiten aprender temas nuevos en salud, generan otras dinámicas para enseñar y aprender, a la vez que estimulan a los niños mediante un aprendizaje divertido. Asimismo, mostraron su interés por seguir empleándolos con sus estudiantes.

Respecto al taller de microscopía, los maestros manifestaron que era posible incluir prácticas pedagógicas similares en sus clases de ciencias. Al mismo tiempo, se les proporcionaron ideas para el desarrollo de estas asignaturas a partir de la actividad lúdica. Por su parte, los escolares hicieron muchas preguntas y se maravillaron al ver los microorganismos. La labor los condujo al descubrimiento de nuevos aprendizajes a través de tareas y mediaciones diferentes.

\section{Aceptación de las actividades lúdicas y toma de conciencia sobre problemas de salud y ambientales}

En general todas las actividades fueron aceptadas gracias a su carácter lúdico y a que tanto niños como adultos a la vez que aprendían, disfrutaban y gozaban lo que estaban haciendo, en particular porque tuvieron la oportunidad de aportar a la adaptación de las mediaciones empleadas. En ningún momento sintieron que las actividades educativas fueron tareas formales sino abiertas, que rompían con lo cotidiano. Las razones de aceptación por cada actividad se reportan en la Tabla 3.

Tabla 3. Razones aceptación actividades lúdicas en La Virgen

\begin{tabular}{|c|c|c|}
\hline Actividades lúdicas & Maestros/otros adultos & Alumnos \\
\hline Capacitaciones & Por metodología y actualización & No constituir una carga académica extra y el disfrute \\
\hline Juegos & Valorados como material educativo & Diversión \\
\hline Títeres & Creatividad y trabajo en grupo & $\begin{array}{l}\text { Diversión, creatividad y motivación por presentarlo en } \\
\text { público. }\end{array}$ \\
\hline Dibujos & $\begin{array}{l}\text { Hacer algo diferente en clase dirigido hacia la salud. } \\
\text { Creación de materiales, como carteleras, utilizando } \\
\text { elementos de la naturaleza. }\end{array}$ & Distracción y diversión \\
\hline $\begin{array}{l}\text { Organización de } \\
\text { Grupos sociales de interés. }\end{array}$ & Apoyo a estudiantes del Grupo JEAR & $\begin{array}{l}\text { Creación del JEAR } \\
\text { JEAR tomó la iniciativa de hacer actividades educativas } \\
\text { lúdicas sobre higiene y parasitismo en la escuela de la } \\
\text { cabecera de la Inspección de La Virgen }\end{array}$ \\
\hline Cartografía Social & $\begin{array}{l}\text { Manifiesta en el préstamo de las instalaciones } \\
\text { educativas para hacer los talleres. }\end{array}$ & $\begin{array}{l}\text { Participaron estudiantes y sus madres. } \\
\text { Alumnos: Gozaron el dibujar y el trabajo cooperativo con sus } \\
\text { madres. } \\
\text { Madres: Les gustó porque hicieron conciencia de los } \\
\text { problemas que los afectan y propusieron posibles soluciones. }\end{array}$ \\
\hline Taller de microscopia & Innovación pedagógica & $\begin{array}{l}\text { "Oportunidad para ver lo que tenemos dentro de nuestro } \\
\text { cuerpo". }\end{array}$ \\
\hline $\begin{array}{l}\text { Diseño, conceptualización y } \\
\text { definición del dispositivo K-kal } \\
\text { para disposición de excretas. }\end{array}$ & $\begin{array}{l}\text { Compartir con la gente en su propio ambiente. } \\
\text { Valoración de sus conocimientos } \\
\text { Disfrute de ludotecarias en la cooperación para la } \\
\text { co-creación del diseño con los niños(as). }\end{array}$ & $\begin{array}{l}\text { Interés en la actividad y propuesta de ideas de diversa } \\
\text { manera. } \\
\text { Trabajo de previo con el equipo de la Universidad Nacional. } \\
\text { Metodología lúdica }\end{array}$ \\
\hline $\begin{array}{l}\text { Carnaval } \\
\text { Frutivegecarnavalito/Factor S. }\end{array}$ & $\begin{array}{l}\text { Apoyo y trabajo colaborativo con las instituciones } \\
\text { participantes. }\end{array}$ & $\begin{array}{l}\text { Por participar en diversas representaciones (mejora de dieta, } \\
\text { vacunación, prevención maltrato infantil y de la pereza) y } \\
\text { actividades (como prevención de las geohelmintiasis). }\end{array}$ \\
\hline
\end{tabular}


Los juegos, que fueron una co-construcción entre la población y el equipo de la Universidad Nacional tuvieron el beneplácito de los usuarios. "Gusarín, Gusarán" gozó de una alta aceptación entre niños y docentes, particularmente de la ludoteca, donde se trabajaba sobre estilos de vida saludables. El otro juego, "Aprender Pescando", según las ludotecarias contó con mayor aceptación que "Gusarín, Gusarán" entre los niños por lo novedoso, especialmente por el uso de cañas de pescar. Es posible que la aceptación de uno u otro juego esté relacionada con la edad de los niños, ya que la ludoteca es esencialmente para los más pequeños. En las escuelas los utilizaron escolares entre cinco a doce años. Es de resaltar que las ludotecarias continuaron empleando los juegos después de terminado el trabajo de campo del grupo de investigación y manifestaron querer participar y colaborar en futuras actividades educativas en pro de la salud con el equipo de trabajo de la Facultad de Medicina de la Universidad Nacional.

En cuanto a la obra de títeres ("El Reino de mi Barriga") es importante señalar que fue re-creada: los niños del casco urbano de La Virgen la representaron de una forma y los de Galilea, bajo la dirección de su profesor, la modificaron de acuerdo a sus expectativas, pero respetando el trasfondo del control de la infección por geohelmintos. Este es un ejemplo de la asimilación y la dinámica propia que van adquiriendo las actividades de educación en salud. La transformación corrobora la postura crítica porque expresa las vivencias de la gente, quien las relaciona con la educación como acto creativo en su vida cotidiana.

Por otra parte, la aceptación del trabajo realizado con la Universidad Nacional se reflejó en el hecho que el Grupo JEAR, por iniciativa propia, escribió y musicalizó dos canciones sobre la relación de las geohelmintiasis con el ambiente. Asimismo, inventaron un bingo para educar y recrear a los escolares sobre temas de salud. Adicionalmente, los niños pequeños de la escuela iniciaron la elaboración de dos canciones.

El taller de cartografía social contó con el apoyo de docentes, lo cual facilitó la participación de escolares con sus madres. Aunque al principio ellas estaban tímidas para dibujar (decían no saber hacerlo), con la motivación de sus hijos y el equipo de la Universidad Nacional, se tranquilizaron y disfrutaron la actividad. Fue así que, según las madres en particular, les sirvió para hacer conciencia sobre problemas ambientales en La Virgen, como: defecar a campo abierto; deficiencias en el manejo de excretas y en el compromiso de las autoridades para solucionarlas; vecinos que no quieren cambiar comportamientos perjudiciales para el ambiente; ausencia de agua apta para consumo y quema de basuras.

El taller de microscopia para maestros y alumnos fue muy bien recibido por varias razones: incluir el material de laboratorio para la identificación de las diferentes formas parasitarias de los geohelmintos y de otros parásitos intestinales también prevalentes en La Virgen como la Giardia intestinalis; la toma de muestras distintas a la materia fecal, como agua y tierra, y la preparación de láminas para la observación microscópica de los huevos y quistes de los principales parásitos intestinales. Dicha actividad se constituyó en un gran descubrimiento para los estudiantes y un estímulo para su interés investigativo.

Respecto al diseño del dispositivo K-kal, fue fundamental la colaboración de los líderes comunitarios, quienes facilitaron el acercamiento a la población y el trabajo casa a casa para identificar las necesidades, gustos y propuestas de la gente. En este proceso fueron claves los recursos lúdicos empleados por el diseñador, tanto para acercar los términos y situaciones técnicos a la población como para ejemplificar físicamente sus ideas mediante el uso de hojas de papel, vegetales, fotos y dibujos. El trabajo con los adultos fue más de conversaciones al calor de tazas de café y de compartir sus actividades diarias, lo cual generó no sólo agrado sino confianza. Durante el diseño del dispositivo mencionado y otras actividades se observó que la gente tenía en sus casas tazas sanitarias, pero no funcionaban; la misma situación se presentaba en algunas escuelas, donde incluso los pozos sépticos tenían fallas en su diseño y el escaso alcantarillado existente desagua en la espesura del bosque.

Los niños también participaron en el diseño del dispositivo K-kal. Con ellos se acudió a la imaginación, los dibujos y el juego creativo (Fotos 1 y 2). Es de resaltar que la permanencia del grupo de la Universidad Nacional en la comunidad fue un factor importante tanto en el acercamiento como en la generación de confianza entre todos los actores involucrados en el proceso y para el conocimiento directo de prácticas culturales. 


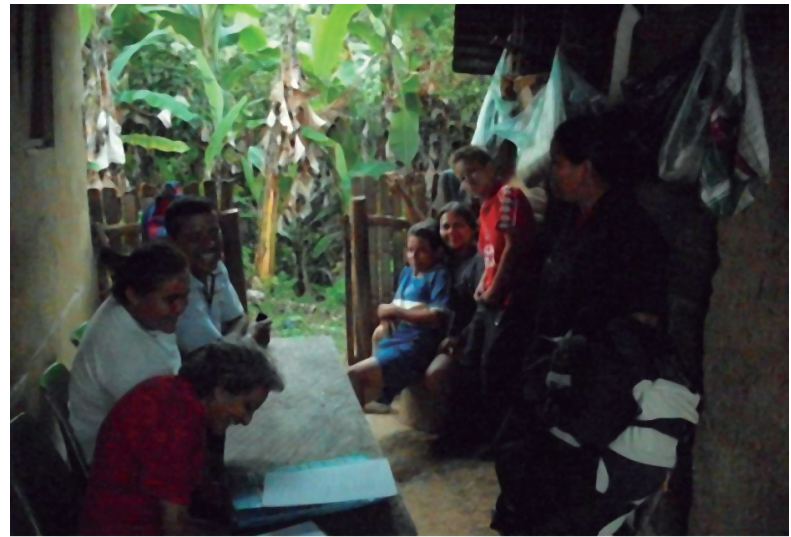

Foto 1. Familia y líder comunitario trabajando en la propuesta del K-kal

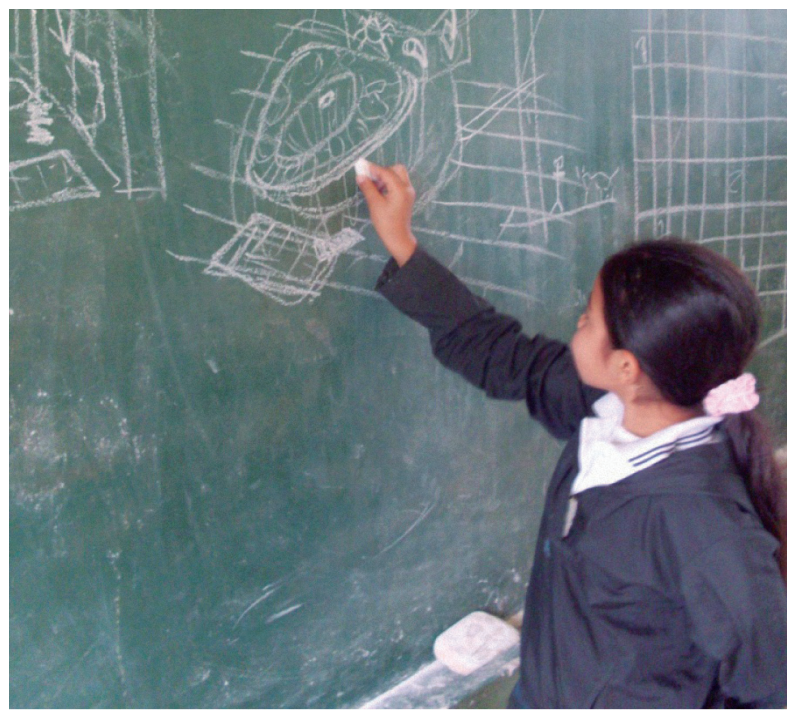

Foto 2. Escolar haciendo propuestas sobre el dispositivo K-kal

Para el cierre de actividades del proyecto se realizó el Festival Frutivegecarnavalito/Factor $S$, que contó con una gran acogida por la población, quien participó masivamente (Fotos 3 a 4). En el carnaval intervino gran parte de la población de La Virgen: a los alumnos de cada escuela de la Inspección se les asignó una representación distinta (por ejemplo: coplas, poesías, canciones, obra de títeres y bailes). Una payasa, invitada por el equipo de la Universidad Nacional, ejecutó, para grandes y chicos, malabares con frutas y verduras; reforzó la relevancia de lavar los alimentos antes de consumirlos, hervir el agua, no andar descalzos, ir al médico, no defecar en el monte y consumir frutas y vegetales en sus comidas diarias. La actividad finalizó con un desfile por el casco urbano de la Inspección, en el que los niños y niñas disfrazados de frutas, verduras, etc. seguían al carro decorado de uno de los líderes comunitarios, daban las gracias a los asistentes y los invitaban a mejorar sus hábitos higiénicos, la alimentación y a reforzar valores positivos que ayuden a la sana convivencia.

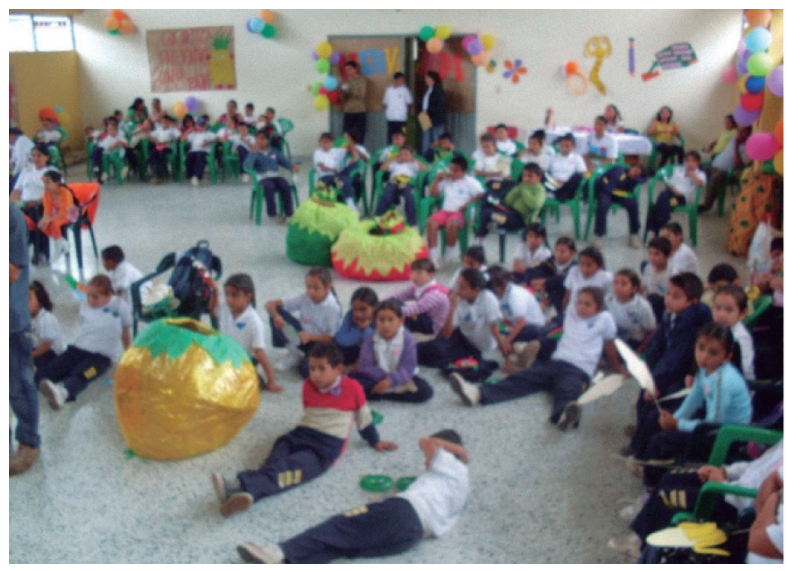

Foto 3. Niños preparándose para el Frutivegecarnavalito/Factor $S$

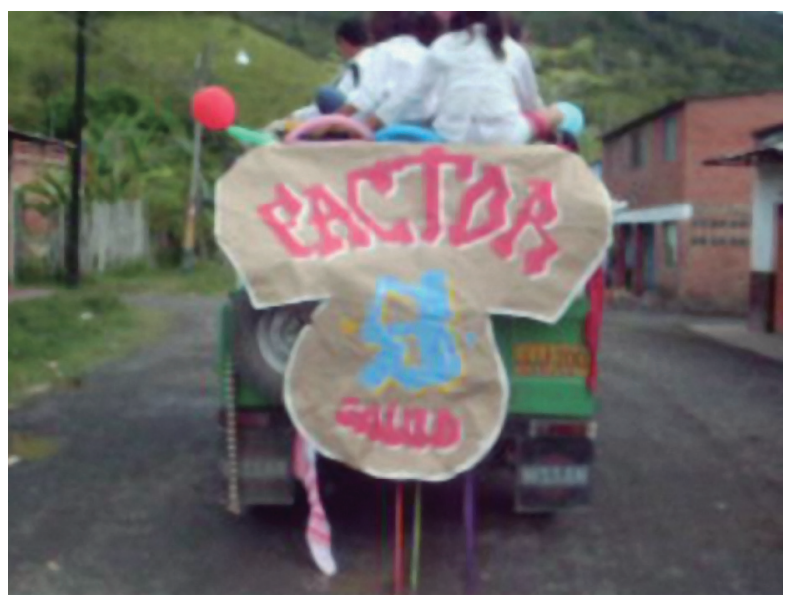

Foto 4. "Carroza" del Factor Salud

\section{Limitantes para la implementación de las actividades lúdicas.}

No hubo muchas limitaciones en la aplicación de las actividades, pero algunas tuvieron mucho peso, como el insuficiente dinero para dar continuidad al proceso y alguna resistencia de los adultos a participar en juegos por considerar que éstos son solo para niños. Por otra parte, los maestros y ludotecarias destacaron algunos obstáculos como suficiente cantidad de juegos, calidad de sus materiales y suficiente personal para acompañar a los niños en el proceso (Tabla 4). 
Tabla 4. Dificultades identificadas en la aplicación de las actividades lúdicas.

\begin{tabular}{lll}
\hline \multicolumn{1}{c}{ Actividad } & \multicolumn{1}{c}{ Maestros/ludotecarias } & \multicolumn{1}{c}{ Investigadores } \\
\hline $\begin{array}{l}\text { Capacitaciones } \\
\text { Juegos }\end{array}$ & $\begin{array}{l}\text { Disponibilidad de juegos. } \\
\text { Resistencia de los materiales de los mismos. } \\
\text { Necesidad de una persona de apoyo para leer las } \\
\text { preguntas de los juegos para darle dinamismo. }\end{array}$ & Mecursos financieros. \\
Cartografía Social & No asistencia de padres hombres porque trabajan. & $\begin{array}{l}\text { Las actividades ligadas a los niños son propias } \\
\text { de las mujeres. }\end{array}$ \\
$\begin{array}{l}\text { Diseño, conceptualización y } \\
\text { definición del dispositivo K-K-kal } \\
\text { para disposición de excretas. } \\
\text { Carnaval }\end{array}$ & - & Ocasionalmente, debilidad en habilidades para \\
Frutivegecarnavalito/Factor S. & - & desarrollar cierto tipo de labores con la gente. \\
\end{tabular}

Valga comentar que esporádicamente la gente no asistió a actividades acordadas con anticipación y a veces se presentaron barreras lingüísticas al pasar del lenguaje técnico al común. Asimismo, en ciertos momentos no se contó con la colaboración de personas que mediaran entre el equipo de trabajo y la población. Tampoco faltaron las circunstancias inesperadas que obligaron a improvisar.

\section{Discusión}

La educación lúdica ambiental optimiza el aprendizaje y permite identificar aspectos relativos a la prevención y al control que deben trabajarse tanto a nivel de saneamiento básico (por ejemplo, ausencia de alcantarillado) como de salud en general. Este tipo de educación mejora la capacidad de argumentación, la relación entre los sujetos que intervienen en el proceso de enseñanza aprendizaje y motiva su creatividad a la par que los faculta para identificar en sí mismos habilidades que antes no reconocían. Además, las actividades lúdicas se constituyen en un medio útil para que la gente haga conciencia de los problemas que afectan su calidad de vida y para plantear posibles soluciones. Actividades lúdicas como los juegos también han sido utilizados en otros lugares de Colombia y en otros países para la prevención y el control del parasitismo intestinal y de otros problemas de salud pública como el dengue $(23-25)$ con resultados e impactos positivos en las poblaciones en las cuales se desarrollaron, tal como sucedió en esta experiencia rural en La Virgen.

La lúdica permite trascender la concepción de educación que la asimila frecuentemente con la información y que suele pretender que las personas eviten casi inmedia- tamente conductas nocivas para su salud. Además, las transformaciones en la calidad de vida de la gente deben partir de producir una reflexión crítica que haga que los individuos se concienticen, que adquieran elementos de análisis que a su vez les permita asumir cambios de conducta, decidir y transformar su realidad abordando aspectos estructurales de la misma, como las relaciones con sus autoridades para lograr mejoras en el saneamiento básico. En ese sentido, la cartografía social es una herramienta que combina lo lúdico con lo crítico para generar conciencia sobre los problemas ambientales de la región.

La educación en salud tiene que trascender las palabras y las reglas para vivir sanamente. Demanda mover emociones y estimular la toma de conciencia sobre la realidad. En ese orden de ideas, la lúdica es una práctica viable y provechosa en los procesos pedagógicos para tal educación, particularmente la relacionada con el control de las geohelmintiasis, pues hace del aprendizaje un proceso dinámico, consciente y gozoso. Por ello, la educación en salud para niños(as) y jóvenes de La Virgen realizada por y para ellos mismos es eficaz pues incrementa la participación comunitaria y se efectúa con pares socioculturales, lo cual genera identificación y trabajo en grupo, como el desarrollado por el Grupo Jóvenes Educadores Aprendiendo a Recrear.

"La participación es una estrategia que permite tanto a los grupos como a las comunidades influir en decisiones que transformen su situación, y así potenciar estrategias de promoción de la salud. La justicia social al igual que la equidad son prerrequisitos para alcanzar mejores niveles de salud y bienestar de las poblaciones; de ahí que alcanzar un ambiente saludable de convivencia requiere la participación 
efectiva de los individuos y colectivos. Es importante que quienes toman las decisiones políticas, al igual que quienes acompañan los procesos en relación con la salud y la calidad de vida tengan presente esta consideración. El gran reto es escuchar a la gente, conocer su situación, sus problemas, así como sus propuestas y estrategias para superarlos. La educación en salud (...) debe dar cuenta de un proceso de acercamiento a la realidad y a la cotidianidad del otro para generar transformaciones, lo que requiere, además de la participación, empoderamiento individual y colectivo." (26).

Por otra parte, cuando se utilizan juegos es importante tener en cuenta que el rumbo y éxito de los mismos está influido por la creatividad y manejo de quien dirige su aplicación (por ejemplo, saber dividir bien los grupos y actuar ante reacciones negativas de los participantes).

\section{Conclusiones y recomendaciones}

Los procesos de infección y reinfección por geohelmintos en los escolares de La Virgen persistirán mientras a dos de las estrategias de control, el tratamiento antihelmíntico (desparasitación) y la educación en salud, no se agregue una mejora definitiva en el saneamiento básico, la otra estrategia clave. La desparasitación, disminuye la morbilidad en los individuos infectados y la educación es esencial para mantener por más tiempo el efecto de la desparasitación y para el control de los procesos de infección y reinfección. Por eso la población debe esforzarse, en conjunto con las autoridades, por proveer a todas las veredas de un sistema adecuado de eliminación de las excretas (como alcantarillado y pozos sépticos) y de agua apta para el consumo humano.

El saneamiento ambiental evita la contaminación del suelo con las formas parasitarias de los geohelmintos. Tales formas (huevos y larvas) son eliminadas en las heces de los individuos infectados cuando defecan en la tierra (campo abierto, "monte", suelo), contaminándola. Igual sucede cuando las personas infectadas hacen sus necesidades cerca de quebradas y ríos, lo cual favorece la contaminación de aguas y de cultivos cuando éstos son regados con esas aguas.

El saneamiento ambiental, por tanto, es considerado como la estrategia más efectiva para disminuir la infección por los geohelmintos en la población. Sin embargo, es fundamental tener en cuenta que las estrategias de control de las geohelmintiasis deben ser integradas: Al menos debe hacerse tratamiento antihelmíntico y educación en salud, pero idealmente acompañadas de saneamiento ambiental para que tengan efectividad a largo plazo.
Dado que las geohelmintiasis como su nombre lo indica son helmintiasis transmitidas por el suelo hay que hacer una intervención sobre éste, pues parte de su ciclo de vida se lleva a cabo en la tierra, en la cual se produce la embrionación, es decir la transformación de formas parasitarias no infectantes a formas parasitarias infectantes. De ahí la importancia de que las poblaciones (gente del común y autoridades) afectadas hagan conciencia de la situación para que generen mecanismos de acción tendientes a disponer de sistemas efectivos de eliminación de excretas para que éstas no contaminen las aguas ni los cultivos. Todo esto representa cambios en los procesos de educación y socialización que incorporen una nueva perspectiva ambiental que mejore la relación del ser humano con el ambiente y se refleje en políticas y planes de desarrollo a nivel local.

Por otra parte, la experiencia mostró que las actividades lúdicas llevadas a cabo con la comunidad son una estrategia adecuada para que la gente identifique problemas ambientales, tome consciencia de ellos y perciba la necesidad de participar en las alternativas de solución. Asimismo, la gente advirtió que fueron actividades vinculadas con su realidad, desarrolladas no como imposiciones externas, sino en respuesta a su contexto socioeconómico y cultural.

En ese mismo sentido, se concluye que los niños aceptan mejor las actividades cuando tienen el carácter lúdico porque se salen de la monotonía de las clases tradicionales y les permite aprender jugando, ser creativos y compartir con los padres de manera recreativa. Además, se constituyen en agentes dinamizadores del proceso educativo, generando constantes cambios en él. Por tanto, se recomienda replicar el proceso, generando las innovaciones necesarias de acuerdo al contexto y recrear los materiales lúdicos en todas las veredas de la Inspección.

Valga agregar que los materiales lúdicos desarrollados y probados en La Virgen pueden ser utilizados en otras poblaciones rurales con características similares, no sólo para prevención y control de las geohelmintiasis sino de otros problemas de salud con sus debidas adecuaciones.

Finalmente, en las actividades se identificaron otros problemas ambientales no relacionados con las geohelmintiasis como el deficiente manejo de basuras, la tala de bosque, el descuido con colillas de cigarrillos que pueden ocasionar incendios y vertimiento de residuos de alambiques. Aspectos que evidenciaron la necesidad de estructurar programas específicos, particularmente uno dirigido al reciclaje, previa capacitación sobre qué se 
puede hacer con los envases plásticos y demás fuentes de basuras o desechos. Sin propuestas concretas, económica y socioculturalmente accesibles no se asegura el éxito de una capacitación para el reciclaje.

\section{Agradecimientos}

A toda la población de La Virgen, en especial a los habitantes de las veredas Galilea y San Nicolás, a Tobías Castiblanco, al profesor Saúl de la escuela de Galilea, a los rectores Guillermo Sánchez y Pedro Martín López y al profesor Daniel León del Colegio Joaquín A. Medina, a Sandra e Ivonne, ludotecarias de Colsubsidio, al Grupo JEAR, a la antropóloga Yolanda Hernández, a las bacteriólogas Carolina Ortiz y Emelina Barraza y al diseñador Edward Geovanny García.

\section{Conflicto de intereses y fuente de financiación}

Los autores declaran no tener conflicto de intereses. Investigación financiada por la División de Investigaciones de la Universidad Nacional de Colombia, Sede Bogotá (DIB).

\section{Referencias}

1. World Health Organization (WHO). Schistosomiasis and Soil Transmitted Helminth Infections. Weekly Epidemiological Record. 2006; 81:145-64.

2. Crompton D W. How much human helminthiasis is there in the world. J Parasitol. 1999;85:397-403.

3. De Silva N R, Brooker S, Hotez P J, Montresor A, Engels D, Savioli L. Soil-transmitted helminth infections: updating the global picture. Trends Parasitol. 2003;19:547-51.

4. Fernández-Niño J A, Reyes-Harker P, Moncada-Alvarez L I, López M C, Cháves M P, Knudson A, Ariza Y. Tendencia y prevalencia de las geohelmintiasis en La Virgen, Colombia 1995-2005. Rev Salud Pública. 2007;9:289-96.

5. Díaz M P, Moncada L I, Reyes P, Fernández J A, Cano D F, Suárez, $\mathrm{R} J$. Conocimientos, actitudes y prácticas sobre las geohelmintiasis en una comunidad rural de Colombia. Revista Med. 2010;8(1):18-28.

6. Gutierrez F, Prado C. Ecopedagogía y ciudadanía planetaria. Ed.Diálogos: Xátiva, 2003.

7. Asaolu S O, Ofoezie I E. The role of health education and sanitation in the control of helminth infections. Acta Tropica. 2003;86:283-94.

8. Nunes de Almeida P. Educación Lúdica, Ediciones San Pablo, Bogotá, s.a. 1994

9. Pilón A F. Desarrollo de la Educación en Salud, Una actualización de conceptos. Rev Saúde Pública. 1986;20:391-6.
10. Jiménez C A, Dinello R, Alvarado L A. Lúdica y Recreación. Texto online: http://www.geocities.com/ludico_pei/ludica_y_recreacion. htm Consultado agosto 22 de 2008.

11. Dinosaurio.com. La importancia del juego en la educación. Texto online: http://www.dinosaurio.com/maestros/la-importancia-del-juego-en-educacion.asp Consultado agosto 22 de 2008.

12. Dias A C, da Silva C V. Tendencias de la producción del conocimiento en la educación en la salud. Rev Latino-am Enfermagem. 2007;15. Texto On line: http://www.scielo.br/pdf/rlae/v15n6/ es 18.pdf Consultado agosto 202008

13. Gutiérrez F, Prieto D. Mediación pedagógica. Apuntes para una educación alternativa. Ediciones La copia Fiel, décima edición. Costa Rica, 2004, p 38.

14. García R, Suárez R, Mateo-de-Acosta O. Comunicación y educación interactiva en salud y su aplicación al control paciente diabético. Rev Panam Salud Publica/Pan Am J Public Health. 1997;2:32-36.

15. Lara M C, Torres S V. A Pedagogical Approach of Schistosomiasis. An Experience in Health Education in Minas Gerais, Brazil. Mem Inst Oswaldo Cruz. 2004;9(1):113-9.

16. Caldeiro GP. Educación problematizadora. 2005. http://freire. idoneos.com/index.php/319078 Educación problematizadora. Consultado 19 Agosto, 2008.

17. Mogensen F. Critical thinking: a central element in developing action competence in health and environmental education. Health Education Research. Theory \& Practice. 1997;12:429-36.

18. Durán E. La estrategia de escuela saludable. Típica Boletín electrónico de salud escolar. 2005;1(1). www.tipica.org Consultado 20 Agosto, 2008.

19. Velásquez J J. Ambientes lúdicos de aprendizaje. Editorial Trillas, México. 2008

20. Hernández R, Fernández C, Baptista P. Metodología de la Investigación. Ed.Mac Graw Hill, México. 2006

21. Normas científicas, técnicas y administrativas para la investigación en salud. Resolución No 008430 de 1993. Santafé de Bogotá. 1993.

22. Códigos del menor. Derechos del niño. Decreto 2737, artículo 13 , noviembre 1989.

23. Rodríguez, A. Protocolo de desparasitación para los escolares. Fundación CIMDER, Cali, Colombia, http://www.col.OPS-OMS.org/ Municipios/Cali/05Desparasitacion.htm, Noviembre 30 de 2002.

24. Toscani N, Duarte A, Leiria L, Tedesco C, Chazan M, Pereira A, Mezzari A. Development and analysis of an educational game for children aiming prevention of parasitological diseases. Interface Comunic., Saúde, Educ. 2007; 11: 281-94.

25. Vivas E, Guevara M. Un juego como estrategia educativa para el control de Aedes aegypti en escolares venezolanos. Rev Panam Salud Publica/Pan Am J Public Health. 2003; 14: 394 - 401.

26. Restrepo S L. La promoción de la salud y sus aportes a la educación en alimentación y nutrición. Invest Educ Enferm. 2005;23(1):110-7. 